\title{
Expression level of NEAT1 differentiates benign and malignant thyroid nodules by regulating NEAT1/miR-9/PTEN and NEAT1/miR-124/PDCD6 signalling
}

\author{
$\mathrm{LI} \mathrm{ZHAO}^{1}$, NA ZHOU ${ }^{2}$ and PING ZHAO ${ }^{3}$ \\ ${ }^{1}$ Department of Ultrasound, Southwest University Hospital, Chongqing 400715; ${ }^{2}$ Department of Abdominal Ultrasound, \\ Xinjiang Autonomous Region Hospital of Traditional Chinese Medicine, Urumchi, Xinjiang 830000; \\ ${ }^{3}$ Department of Ultrasound, Shangluo Central Hospital, Shangluo, Shaanxi 726000, P.R. China
}

Received July 8, 2019; Accepted May 12, 2020

DOI: $10.3892 / \mathrm{ijmm} .2020 .4721$

\begin{abstract}
The incidence of thyroid nodules has been increasing worldwide; however, there are currently no feasible and robust methods to differentiate malignant thyroid nodules from benign thyroid nodules. The present study aimed to establish a practical method to determine the malignancy of thyroid nodules. Reverse transcription-quantitative PCR and western blot analyses were performed to compare the levels of long non-coding RNA nuclear enriched abundant transcript 1 (NEAT1), microRNA (miR)-9, miR-124, PTEN and programmed cell death protein 6 (PDCD6) in the peripheral blood and thyroid tissue samples between patients with malignant and benign thyroid nodules. Additionally, a regulatory relationship between NEAT1, miR-124, miR-9, PTEN and PDCD6 was established in the present study. The diagnostic value of NEAT1, miR-124 and miR-9 was determined using a ROC analysis. The expression levels of NEAT1, PTEN and PDCD6 in peripheral blood and thyroid tissue samples collected from the benign group were higher compared with those in the malignant group, whereas the expression levels of miR-124 and miR-9 were lower in the benign group. In the peripheral blood, NEAT1 expression exhibited an area under the curve (AUC) value of 0.8546, whereas miR-124 and miR-9 expression had AUC values of 0.7657 and 0.7019 , respectively. In the thyroid tissue, NEAT1, miR-124, and miR-9 had AUC values of $0.9304,0.8221$ and 0.7757 , respectively. Additionally, miR-9 and miR-124 expression levels in BCPaP and SW579 cells was decreased after transfection with a NEAT1 expression vector compared with those in cells transfected with the control vector, whereas the expression of PTEN and PDCD6
\end{abstract}

Correspondence to: Professor Ping Zhao, Department of Ultrasound, Shangluo Central Hospital, 148 Beixin Street, Shangzhou, Shangluo, Shaanxi 726000, P.R. China

E-mail: ultranodule@outlook.com

Key words: thyroid nodule, apoptosis, NEAT1, microRNA-9, PTEN, microRNA-124, programmed cell death protein 6 was upregulated. By contrast, transfection with short hairpin RNA targeting NEAT1 notably increased the expression of miR-9 and miR-124 while downregulating the expression of PTEN and PDCD6 compared with that in the control cells. In conclusion, the results of the present study demonstrated that the dysregulation of NEAT1 expression may be used to differentiate benign and malignant thyroid nodules.

\section{Introduction}

Common thyroid nodules are usually benign $(1,2)$. However, according to studies on fine-needle aspiration cytology, among the nodules identified through clinical examinations, $\sim 15 \%$ of the nodules are malignant $(3,4)$. Correspondingly, the identification of a thyroid nodule with a diameter of $\geq 1 \mathrm{~cm}$ frequently prompts the initialization of a more thorough diagnostic evaluation (5). Fine-needle aspiration is the key method for the evaluation of thyroid nodules, enabling the evaluation of cellular morphology features that cannot be identified through clinical imaging or assessment (6). Preoperative fine-needle aspiration-guided ultrasonography has been indicated to accurately identify $\leq 80 \%$ of all thyroid nodules, thus avoiding surgery for diagnosis (7).

According to genome-wide sequencing results, non-coding genes account for up to $\leq 98 \%$ of the whole genome (8). Among these non-coding genes, long non-coding RNAs (lncRNAs), which are $>200 \mathrm{nt}$ long, exert crucial effects on different cellular processes and are dysregulated under abnormal conditions, including rare diseases such as cat eye, Klinefelter's and Down's syndromes $(9,10)$. In addition, lncRNAs modulate the expression of genes through multiple mechanisms, such as miRNA degradation, epigenetic modification, transcription control and gene imprinting $(11,12)$. Other types of non-coding RNAs include microRNAs (miRNAs), which are $22 \mathrm{nt}$ long and repress the expression of genes under various biological conditions by binding to the 3'-untranslated regions (UTRs) of target mRNAs and by triggering the degradation or suppressing the translation of mRNAs (13).

LncRNAs are involved in tumourigenesis. For example, the expression of IncRNA nuclear enriched abundant transcript 1 (NEAT1) is significantly enhanced in cervical cancer 
cell lines and tissues $(14,15)$. In a molecular study, NEAT1 has been demonstrated to function as a competing endogenous RNA (ceRNA) by repressing miR-9-5p expression; in addition, high levels of NEAT1 expression reduced the inhibitory effect of miR-9-5p on the expression of POU class 2 transcription factor 1 (POU2F1) and PTEN (16). Consistent with the observation that NEAT1 negatively regulated miR-9-5p, the upregulation of miR-9-5p suppressed NEAT1-induced cancer cell proliferation $(17,18)$. The expression of miR-9 was also observed to be negatively associated with the expression of PTEN, a tumour suppressor gene that was confirmed as a direct target gene of miR-9, in laryngocarcinoma cells, and the introduction of miR-9 mimics into laryngocarcinoma cells facilitated cell metastatic capability and proliferation (19). In nasopharyngeal carcinoma (NPC) cells and tissues, NEAT1 was also upregulated, whereas miR-124 was downregulated; knockdown of NEAT1 suppressed NPC progression and facilitated apoptosis, whereas overexpression of NEAT1 resulted in the opposite outcome (20). Furthermore, NEAT1 was confirmed to inhibit the expression of miR-124 through direct interaction in NPC cells (20). In addition, the anti-apoptotic and pro-proliferative effects mediated by NEAT1 were reversed by miR-124 (20).

Using bioinformatics methods and in vitro experiments, programmed cell death protein 6 (PDCD6) was identified as a protein directly targeted by miR-124-3p (21). The restoration of the expression of PDCD6 impaired the suppressive effect of miR-124-3p on metastasis by facilitating the infiltration of cancer cells (21). In addition, miR-124-3p expression levels were inversely related to the levels of PDCD6 mRNA in clinical samples of breast tumours (21). Therefore, these results suggested that miR-124-3p suppressed the metastasis of tumours by repressing the expression of PDCD6, and that the miR-124-3p/PDCD6 signalling axis may be a target for new treatments for patients with advanced breast cancer (BC) (21).

Deregulated miR-9 and miR-124 have been used as biomarkers to differentiate benign and malignant thyroid nodules (22). Previous studies have reported that NEAT1 may function as a sponge of miR-9 and miR-124 $(16,20)$. In addition, PTEN and PDCD6 have also been identified as possible targets of miR-9 and miR-124, respectively. PTEN and PDCD6 have been implicated in tumourigenesis (23-25). In the present study, peripheral blood and thyroid tissue samples were collected to investigate the roles of NEAT1/miR-9/PTEN and NEAT1/miR-124/PDCD6 signalling in the differentiation of benign and malignant thyroid nodules, and to establish a practical method to determine the malignancy of thyroid nodules.

\section{Materials and methods}

Human subjects and sample collection. A total of 98 patients diagnosed with thyroid nodules between August 2014 and March 2017 at Shangluo Central Hospital (Shangluo, China) were enrolled in the present study. The subjects included 52 patients with malignant thyroid nodules (MTN or the malignant group; nodule size range, $2.03-3.63 \mathrm{~cm}$ ) and 46 patients with benign thyroid nodules (BTN or the benign group; nodule size range, 2.06-3.46 cm). Peripheral blood and thyroid tissue samples were collected from all participants. The demographic and clinicopathological characteristics of the participants, such as age, sex, nodule size, solitary nodule, free triiodothyronine (FT3), free thyroxine (FT4) and thyroid-stimulating hormone (TSH) levels, were recorded and analysed. The inclusion criteria of the participants were as follows: i) 18-70 years old without gender restrictions; ii) confirmed diagnosis of thyroid nodules by imaging and histological examinations; iii) not received medication that may have influenced the metabolism of the thyroid in the previous three months; and iv) no diseases that influence the metabolism of the thyroid, including chronic thyroid dysfunction. The protocol of the present study was approved by the Ethics Committee of Shangluo Central Hospital, and all subjects signed informed consent forms.

$R N A$ isolation and reverse transcription-quantitative PCR $(R T-q P C R)$. To establish the accuracy of the microRNA assays, RT-qPCR was used to confirm the differential miRNA expression in the samples. Total RNA was isolated from the collected samples and cells using TRIzol ${ }^{\circledR}$ reagent (Invitrogen; Thermo Fisher Scientific, Inc.), and the purification was performed using the Qiagen RNeasy mini kit (Qiagen $\mathrm{GmbH}$ ). RNA concentration was measured by a spectrophotometer. Reverse transcription with the cDNA Synthesis kit (Invitrogen; Thermo Fisher Scientific, Inc.) and qPCR (PowerUp SYBR ${ }^{\circledR}-$ Green; Thermo Fisher Scientific, Inc.) were performed using an ABI real-time PCR System (Applied Biosystems; Thermo Fisher Scientific, Inc.). The cDNA synthesis was performed according to the manufacturer's instructions. The thermocycling conditions of the qPCR were as follows: $95^{\circ} \mathrm{C}$ for $10 \mathrm{~min}$, followed by 40 cycles of $95^{\circ} \mathrm{C}$ for $15 \mathrm{sec}$ and $60^{\circ} \mathrm{C}$ for $60 \mathrm{sec}$. U6 (for miRNA) and GAPDH (for mRNA) were used as the internal control to calculate the normalized relative expression levels of NEAT1, miR-124, miR-9, PTEN and PDCD6 mRNA using the $2^{-\triangle \Delta C q}$ method (26). Primer Premier 5.0 (Premier Biosoft) was used to design primers for qPCR. The primers used were as follows: NEAT1 forward, 5'-GCTGGA CCTTTCATGTAACGGG-3' and reverse, 5'-TGAACTCTG CCGGTACAGGGAA-3'; miR-124 forward, 5'-TTCACAGCG GACCTTGA-3' and reverse, 5'-GAACATGTCTGCGTATCT C-3'; miR-9 forward, 5'-TCTTTGGTTATCTAGCTGT-3' and reverse, 5'-GAACATGTCTGCGTATCTC-3'; PTEN forward, 5'-TGAGTTCCCTCAGCCGTTACCT-3' and reverse, 5'-GAG GTTTCCTCTGGTCCTGGTA-3'; PDCD6 forward, 5'-ACA TCACGGACTGGCAGAACGT-3' and reverse, 5'-AGGATG TCGTGGAACTGGTCAG-3'; U6 forward, 5'-CTCGCTTCG GCAGCACA-3' and reverse, 5'-AACGCTTCACGAATT TGCGT-3'; and GAPDH forward, 5'-GTCTCCTCTGACTTC AACAGCG-3' and reverse, 5'-ACCACCCTGTTGCTGTAG CCAA-3'.

Cell culture and transfection. BCPaP and SW579 cells were obtained from the Shanghai Cell Bank of the Chinese Academy of Sciences. All cells were routinely cultured in DMEM (Gibco; Thermo Fisher Scientific, Inc.) containing 10\% FBS in an incubator with $5 \% \mathrm{CO}_{2}$ at $37^{\circ} \mathrm{C}$. When the cells were $80 \%$ confluent, they were transfected with $30 \mathrm{nM}$ sh-NEAT1 (Guangzhou RiboBio Co., Ltd.), scramble control (Guangzhou RiboBio Co., Ltd.), pcDNA3.1-NEAT1 overexpression vector (p-lncRNA-NEAT1; Shanghai GenePharma Co., Ltd.) or pcDNA3.1 empty vector (p-vector; Shanghai GenePharma Co., 
Ltd.) using Lipofectamine ${ }^{\circledR} 2000$ (Invitrogen; Thermo Fisher Scientific, Inc.) according to the manufacturer's instructions for $48 \mathrm{~h}$ at room temperature. At $48 \mathrm{~h}$ post-transfection, the expression levels of NEAT1, miR-9 and miR-124 in the transfected cells were measured by RT-qPCR.

Western blotanalysis.Protein was extracted from the whole-cell lysate using RIPA buffer (Cell Signaling Technology, Inc.). Subsequently, western blotting was performed using a routine method. Briefly, the protein concentration was measured with a BCA assay kit (Bio-Rad Laboratories, Inc.), and $50 \mu \mathrm{g} /$ lane of protein was resolved by a $10 \%$ Mini-PROTEAN TG gel (Bio-Rad Laboratories, Inc.) and loaded onto a PVDF membrane. The membranes were blocked using a Tris buffer containing 5\% skim milk and $0.1 \%$ Tween-20 for $60 \mathrm{~min}$ at room temperature. Subsequently, the membrane was incubated overnight at room temperature with anti-PDCD6 $(1: 1,000$; cat no. ab109181; Abcam) and anti-PETN (1:1,000; cat no. ab267787; Abcam) primary antibodies, washed with a Tris buffer containing $0.1 \%$ Tween-20 three times and incubated with an HRP-conjugated secondary antibody $(1: 2,000$; cat no. ab6721; Abcam) at room temperature for $1 \mathrm{~h}$. The gel was visualized using Pierce ${ }^{\mathrm{TM}}$ ECL Western Blotting Substrate (cat. no. 32109; Thermo Fisher Scientific, Inc.) and analysed by ImageJ $1.44 \mathrm{e}$ software (National Institutes of Health). Anti- $\beta$-actin (1:1,000; cat. no. MA5-15739; Invitrogen; Thermo Fisher Scientific, Inc.) was used as the internal control to calculate the relative expression levels of PDCD6 and PTEN proteins.

Immunohistochemistry. The expression of PDCD6 and PTEN in paraffin-embedded thyroid nodule tissues was determined using immunohistochemistry. Briefly, 4- $\mu \mathrm{m}$ sections were prepared from representative paraffin-embedded specimens of thyroid nodule tissues. Surrounding normal thyroid nodule tissues were used as the control. Xylene was used to deparaffinize the sections twice, and five gradient ethanol baths (100, $95,85,75$ and $60 \%$ ) were used to rehydrate the resultant sections. Antigen retrieval was performed by heating the sections for $15 \mathrm{~min}$ in a pressure cooker, and EDTA buffer ( $\mathrm{Ph} \mathrm{8.0)}$ was added prior to incubation with $3 \% \mathrm{H}_{2} \mathrm{O}_{2}$ for 10 min to block endogenous peroxidase activity. Subsequently, 1X PBS (Gibco; Thermo Fisher Scientific, Inc.) was used to wash the sections, and the sections were incubated with primary anti-PDCD6 (cat. no. ab9181; 1:1,000 dilution) and anti-PTEN (cat. no. ab267787; 1:1,000 dilution; both from Abcam) antibodies was performed overnight at $4^{\circ} \mathrm{C}$. Horseradish peroxidase-labelled anti-rabbit IgG secondary antibodies (cat. no. ab6721; 1:2,000 dilution; Abcam) were used to treat samples for $30 \mathrm{~min}$ at room temperature. 3,3'-Diaminobenzidine was used to develop the sections, which were counterstained with haematoxylin and observed under an Olympus light microscope (magnification, x200; Olympus Corporation).

Statistical analysis. Data are presented as the mean \pm SD. P-values and fold-changes were used to evaluate the differences in mRNA, miRNA and lncRNA expression between different groups. Receiver operating characteristic (ROC) curves were generated to evaluate the diagnostic values of NEAT1, miR-124 and miR-9 for malignant thyroid nodules by analysing the area under the curve (AUC) of the candidate genes. The intergroup differences were analysed with unpaired Student's t-test using SPSS version 16.0 software (SPSS, Inc.). The $\chi^{2}$ test was used for the analysis of characteristic distribution of the patients enrolled in this study. $\mathrm{P}<0.05$ was considered to indicate a statistically significant difference.

\section{Results}

Demographic, clinicopathological and genotypic parameters of the study participants. The demographic and clinicopathological characteristics of the 98 participants diagnosed with thyroid nodules are summarized in Table I. The majority of the MTN or BTN subjects were female, aged between 42 and 60 years. In addition, parameters including nodule size, solitary nodule, FT3, FT4 and TSH were also compared between the two groups; no significant differences in these parameters were observed between the MTN and BTN groups.

Differential expression of NEAT1, miR-9 and miR-124. RT-qPCR was performed to compare the levels of NEAT1, miR-9 and miR-124 in the peripheral blood and thyroid tissue samples between the MTN and BTN groups. The NEAT1 levels in the peripheral blood (Fig. 1A) and thyroid tissue (Fig. 2A) samples of the BTN group were higher compared with those in the MTN group, whereas lower levels of miR-124 (Figs. 1B and 2B) and miR-9 (Figs. 1C and 2C) were detected in the BTN group, suggesting that malignant thyroid nodules were associated with the downregulation of NEAT1 and upregulation of miR-124/miR-9.

Differential expression of PTEN and PDCD6. RT-qPCR and IHC were performed to compare the levels of PTEN and PDCD6 in the peripheral blood and thyroid tissue samples between the MTN and BTN groups. An evident increase in the mRNA (Fig. 3A) and protein (Fig. 3B) levels of PTEN was observed in thyroid tissues collected from the BTN group compared with those from the MTN group, along with higher mRNA (Fig. 4A) and protein (Fig. 4B) levels of PDCD6, suggesting that malignant thyroid nodules were associated with downregulation of PTEN and PDCD6 expression.

Diagnostic values of NEAT1, miR-124 and miR-9 for malignant thyroid nodules. ROC analysis was performed to evaluate the diagnostic values of NEAT1, miR-124 and miR-9 for malignant thyroid nodules. In the peripheral blood samples, NEAT1, miR-124 and miR-9 exhibited AUC values of 0.8946, 0.7657 and 0.7019 , respectively (Fig. 5A). In thyroid tissue samples, NEAT1, miR-124 and miR-9 had AUC values of $0.9304,0.8221$ and 0.7757 , respectively (Fig. 5B).

The regulatory relationship among NEAT1, miR-124, miR-9, PTEN and PDCD6. RT-qPCR and western blot analyses were performed to explore the regulatory relationship among NEAT1, miR-124, miR-9, PTEN and PDCD6. The levels of miR-124, miR-9, PTEN and PDCD6 in BCPaP and SW579 cells were determined following transfection with NEAT1 constructs. As presented in Fig. 6, miR-9 (Fig. 6A) and miR-124 (Fig. 6B) levels in BCPaP and SW579 cells were 
Table I. Characteristics of the participants of the present study.

\begin{tabular}{lccc}
\hline Characteristics of patients & BTN $(\mathrm{N}=46)$ & MTN (N=52) & P-value \\
\hline Sex, male (\%) & $9(19.6)$ & $11(21.2)$ & $50.6 \pm 7.4$ \\
Age, years, mean \pm SD & $51.3 \pm 8.9$ & $2.76 \pm 0.7$ & 0.685 \\
Nodule size, cm, mean \pm SD & $2.83 \pm 0.8$ & $21(40.4)$ & 0.788 \\
Solitary nodule, n (\%) & $18(39.1)$ & $4.4(3.8-5.1)$ & 0.453 \\
FT3, pmol/l, median (IQR) & $4.6(3.7-5.3)$ & $15.5(14.3-16.9)$ & 0.748 \\
FT4, pmol/l, median (IQR) & $15.3(13.3-17.6)$ & $1.2(0.5-2.1)$ & 0.957 \\
TSH, mIU/ml, median (IQR) & $1.2(0.6-2.1)$ & 0.994
\end{tabular}

BTN, benign thyroid nodule; MTN, malignant thyroid nodule; FT3, free triiodothyronine; FT4, free thyroxine; TSH, thyroid-stimulating hormone; IQR, interquartile range.

markedly downregulated following transfection with the NEAT1 overexpression construct compared with those in the corresponding negative control groups, along with markedly upregulated PTEN mRNA (Fig. 6C) and protein (Fig. 6E) expression. In addition, BCPaP and SW579 cells transfected with the NEAT1 overexpression construct exhibited higher mRNA (Fig. 6D) and protein (Fig. 6F) levels of PDCD6 compared with those in cells transfected with the negative control vector. The transfection of BCPaP and SW579 cells with sh-NEAT1 inhibited NEAT1 expression (Fig. 7A), along with increased levels of miR-9 (Fig. 7B) and miR-124 (Fig. 7C) compared with those in the negative control groups. In addition, the transfection of BCPaP and SW579 cells with sh-NEAT1 substantially downregulated the expression of PTEN mRNA (Fig. 7D) and protein (Fig. 7F), as well as the expression of PDCD6 mRNA (Fig. 7E) and protein (Fig. 7G) compared with those in the negative control groups. The successful transfections of NEAT1 overexpression vector and sh-NEAT1 were verified by the evidently upregulated (Fig. S1A) and downregulated (Fig. 1B) expression of NEAT1, respectively.

\section{Discussion}

It is essential to identify an approach to effectively differentiate benign nodules from thyroid carcinoma as they are difficult to distinguish using fine-needle aspiration, leading to unnecessary thyroidectomy (26). The present study compared the levels of NEAT1, miR-9 and miR-124 in the peripheral blood and thyroid tissue samples collected from subjects with malignant and benign thyroid nodules. The results demonstrated that NEAT1 expression was significantly higher in the peripheral blood and thyroid tissue samples of the BTN group compared with that in samples from the MTN group, whereas the expression levels of miR-124 and miR-9 were lower in the BTN group. The levels of PTEN and PDCD6 expression in the peripheral blood and thyroid tissues of the BTN group were higher compared with those in the tissues from the MTN group. Previous studies have demonstrated that, compared with patients with benign nodules, the expression of circulating miR-5691, miR-9-3p and miR-124-3p was increased in patients with papillary thyroid cancer (PTC), along with decreased levels of miR-196b-5p and miR-4701 (22). The abnormalities in the regulation of miR-196b-5p, miR-4701, miR-9-3p and miR-124-3p were further confirmed using RT-qPCR; the levels of miR-9-3p and miR-124-3p were increased in patients with PTC compared with those of other candidate miRNAs (22). Another previous study demonstrated that miR-124-3p and miR-9-2p could differentiate between benign and malignant nodules with high specificity and sensitivity compared with other candidate miRNAs (26), which was consistent with the results of the present study. In addition, the present study identified that the IncRNA NEAT1 regulated the expression of multiple miRNAs by sponging them, and the results also demonstrated that, compared with the other studied candidate genes, NEAT may be a more accurate biomarker for the differentiation between malignant and benign thyroid nodules with higher sensitivity and specificity.

PTEN is involved in the regulation of metastasis, apoptosis, proliferation and cell cycle progression, and low PTEN expression has been reported to be associated with the progression and development of human pharyngeal squamous cell cancer $(27,28)$. A previous study reported that PTEN acted as a downstream target gene of miR-9 and functioned as a mediator of miR-9 in laryngocarcinoma cells, which suggested that miR-9 could bind to the PTEN 3'-UTR to inhibit its transcription (19). Another study demonstrated that miR-9 modulated PTEN expression at the posttranscriptional level (19). PTEN has been reported to act as a cancer suppressor gene modulating the PTEN/PI3K/AKT/mTOR signalling pathway, which is often dysregulated in breast and prostate cancer (29). Therefore, by modulating the miR-9/PTEN signalling axis, NEAT1 may participate in the differentiation of benign and malignant thyroid nodules.

PDCD6 is a $\mathrm{Ca}^{2+}$-binding protein with a molecular weight of $22 \mathrm{kDa}$ and an open reading frame encoding a sequence of 191 amino acids (31). In addition, PDCD6 has five EF-hand-like motifs that are serially repetitive and form a region comprising $\mathrm{Ca}^{2+}$-binding domains (32). The mRNA expression of PDCD6 is ubiquitous among various tissues in mammals, and this pattern of expression is associated with the process of apoptosis (31). In lower eukaryotes (such as Dictyostelium discoideum), plants and insects, proteins resembling PDCD6 have also been observed and identified to function similarly to their homologs in mammals (33). The mRNA and protein levels 
A

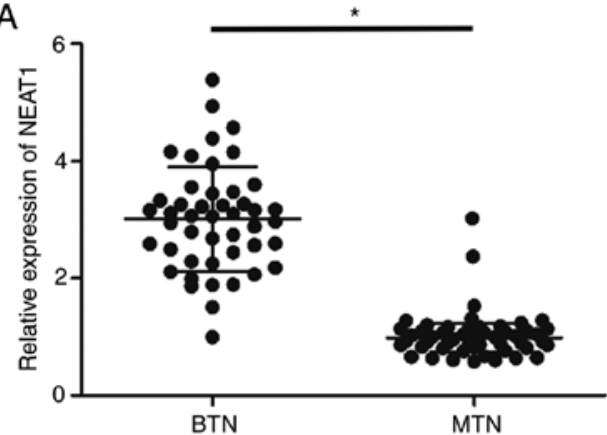

B

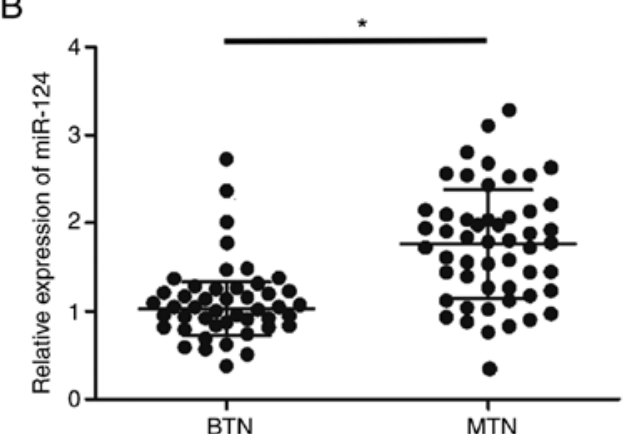

C

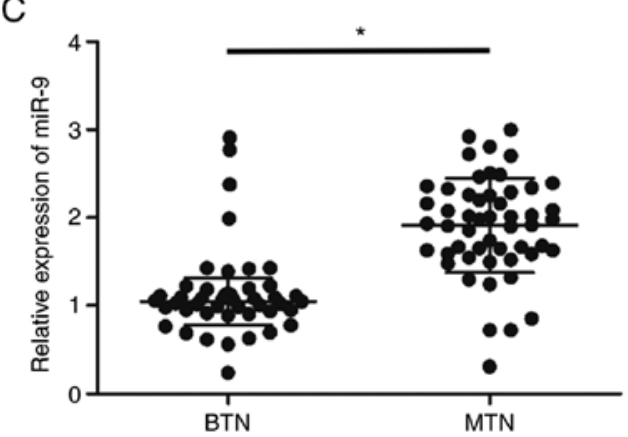

Figure 1. Differential expression of NEAT1, miR-9 and miR-124 in peripheral blood samples between the MTN and BTN groups. (A) NEAT1 was more highly expressed in peripheral blood samples of the BTN group compared with that in the MTN group. (B) miR-124 was expressed at lower levels in peripheral blood samples from the BTN group compared with those in the MTN group. (C) miR-9 was expressed at lower levels in peripheral blood samples from the BTN group compared with those in the MTN group. ${ }^{*} \mathrm{P}<0.05$. BTN, benign thyroid nodule; MTN, malignant thyroid nodule; NEAT1, nuclear enriched abundant transcript 1; miR, microRNA.

of PDCD6 are high in metastatic ovarian cancer as well as in mesenchymal tumours $(34,35)$. According to a previously published study by Zhang et al (21), the role of miR-124-3p in BC metastasis is regulated by the suppression of PDCD6. A functional correlation between PDCD6 and miR-124 in primary tumours has been indicated by the characterization of the miR-124-3p/PDCD6 signalling axis in patients with BC (21). Therefore, NEAT1 may interfere with the differentiation of benign and malignant thyroid nodules, possibly via the miR-124/PDCD6 signalling axis.

Previous studies have indicated that the expression of NEAT1 is upregulated in various types of human cancer, such as leukaemia, lung cancer and gastric carcinoma $(17,18)$. NEAT1 upregulation facilitates the malignancy of stomach tumours (17). In addition, the oncogenic effect of NEAT1 has also been observed in $\mathrm{BC}(36,37)$. Han et al (38) also reported that NEAT1 enhanced the resistance of cervical cancer to
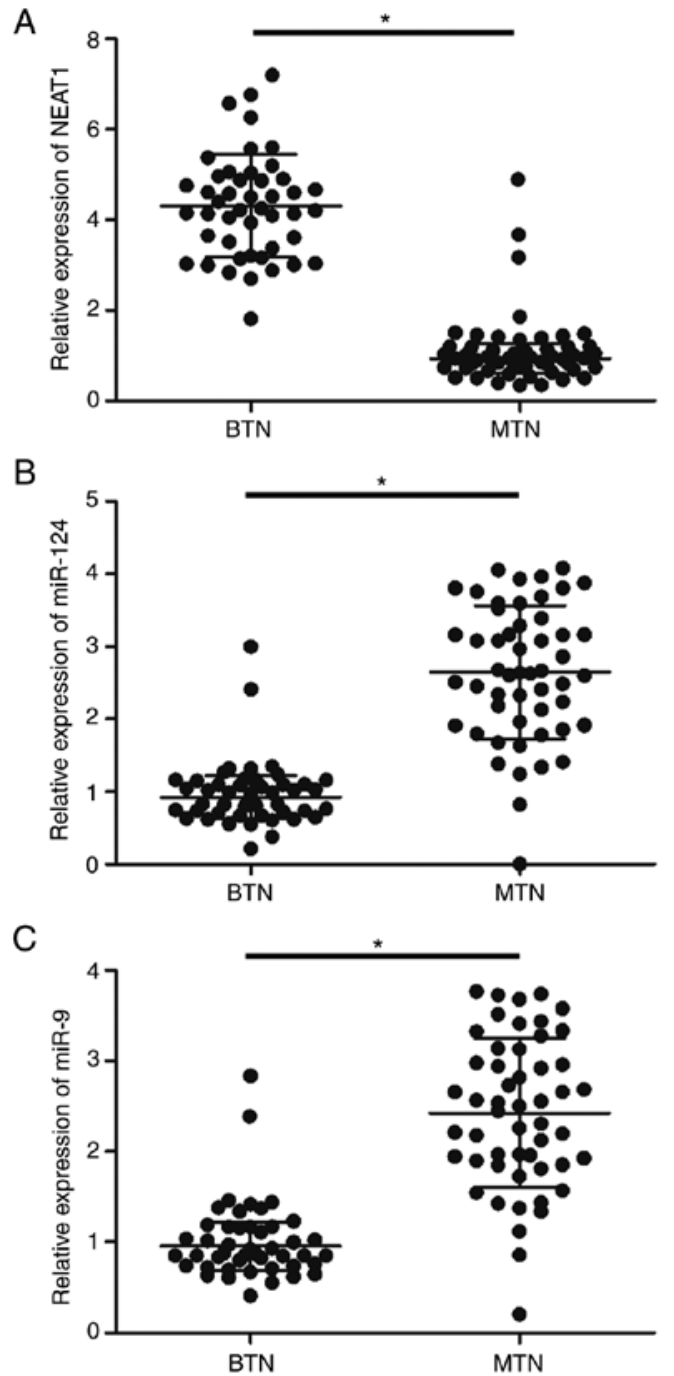

Figure 2. Differential expression of NEAT1, miR-9, and miR-124 in thyroid tissues. (A) NEAT1 levels in thyroid tissues of the BTN group were higher compared with those in the MTN group. (B) miR-124 levels in thyroid tissues of the BTN group were lower compared with those in the MTN group. (C) miR-9 levels in thyroid tissues of the BTN group were lower compared with those in the MTN group. "P<0.05. BTN, benign thyroid nodule; MTN, malignant thyroid nodule; NEAT1, nuclear enriched abundant transcript 1; miR, microRNA.

radiation by regulating miR-193b-3p/cyclin D1 signalling. Overexpression of NEAT1 was observed to promote cervical cancer cell proliferation, and NEAT1 acted as a ceRNA to negatively regulate miR-9-5p expression; the levels of the downstream targets of miR-9-5p such as POU2F1 and PTEN were accordingly elevated following NEAT1 overexpression (16). Upregulation of miR-9-5p could reverse the NEAT1-facilitated cancer cell proliferation, suggesting that miR-9-5p may be a target molecule of NEAT1 in a signalling cascade associated with cervical cancer progression (16). It was also observed that NEAT1 was located in the interchromatin space of the nucleus (39). Previous studies have reported that NEAT1 is an oncogene in a number of types of cancer such as lung, oesophageal and colorectal cancer as well as hepatocellular carcinoma (40). Of note, radioimmunoprecipitation assays also confirmed that NEAT1 targeted miR-124 and was able to inhibit the expression of miR-124 (41). A previous study also 

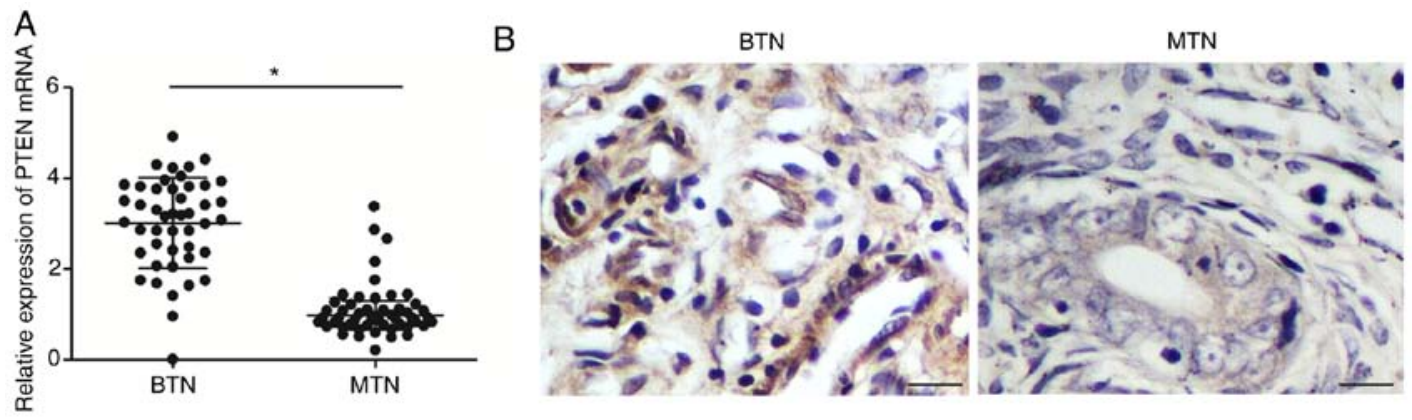

Figure 3. Differential expression of PTEN in thyroid tissues was determined using reverse transcription-quantitative PCR and immunohistochemistry. (A) The PTEN mRNA expression in thyroid tissue samples of the BTN group was higher compared with that in the MTN group. (B) The PTEN protein expression in thyroid tissue samples of the BTN group appeared higher compared with that in the MTN group. Scale bar, $100 \mu \mathrm{m}$. "P<0.05 vs. BTN. BTN, benign thyroid nodule; MTN, malignant thyroid nodule.

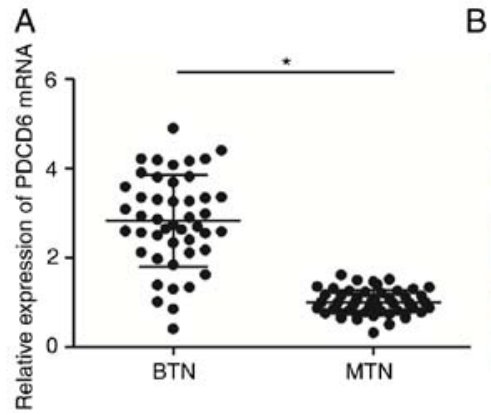

B

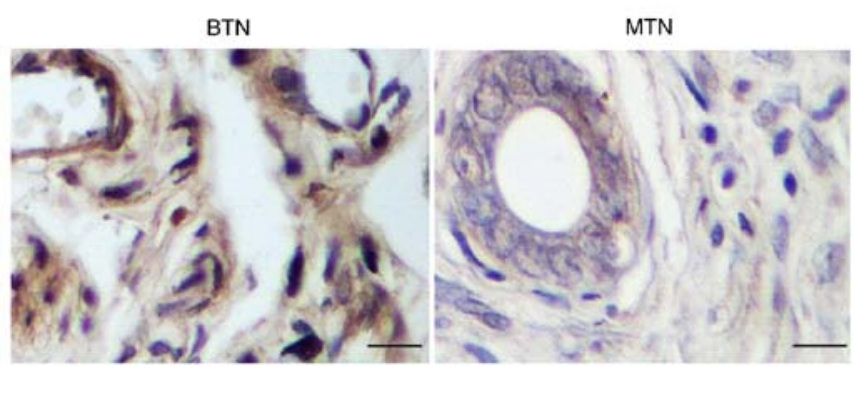

Figure 4. Differential expression of PDCD6 in thyroid tissues was determined using reverse transcription-quantitative PCR and immunohistochemistry (A) The PDCD6 mRNA expression in thyroid tissue samples of the BTN group was higher than compared with that in the MTN group. (B) The PDCD6 protein expression in thyroid tissue samples of the BTN group appeared higher compared with that in the MTN group. Scale bar, $100 \mu \mathrm{m}$. "P<0.05 vs. BTN. BTN, benign thyroid nodule; MTN, malignant thyroid nodule; PDCD6, programmed cell death protein 6.
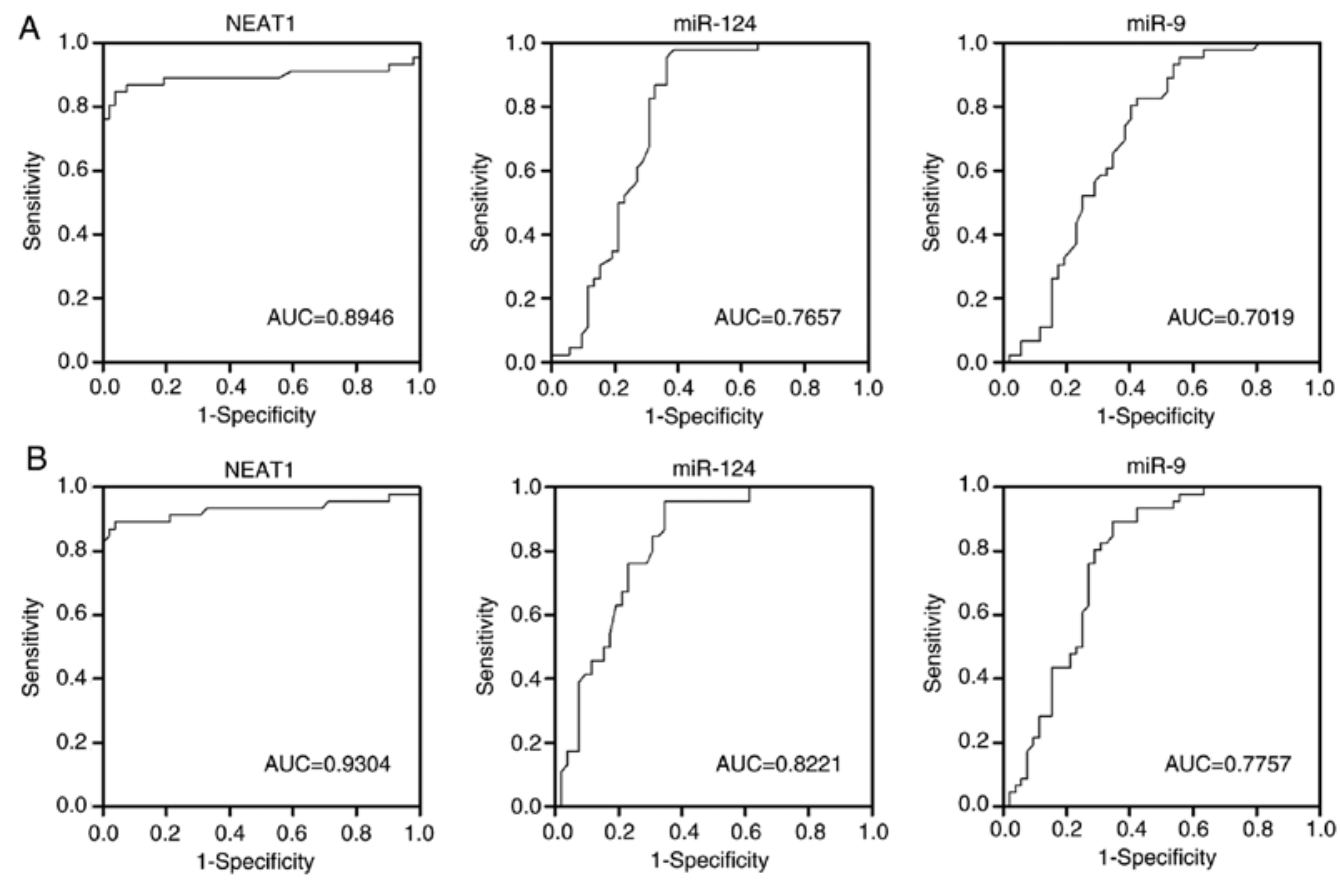

Figure 5. ROC analysis of the diagnostic values of NEAT1, miR-124 and miR-9 for malignant thyroid nodules. (A) NEAT1, miR-124 and miR-9 ROC curves using peripheral blood samples. (B) NEAT1, miR-124 and miR-9 ROC curves using thyroid tissue samples. NEAT1, nuclear enriched abundant transcript 1; miR, microRNA; ROC, receiver operating characteristic; AUC, area under the curve.

demonstrated a correlation between NEAT1 and miR-124-3p expression levels in ovarian cancer cells (41). However, a contradictory statement was published by Idogawa et al (42); in their study, lncRNA NEAT1 was identified as a direct 

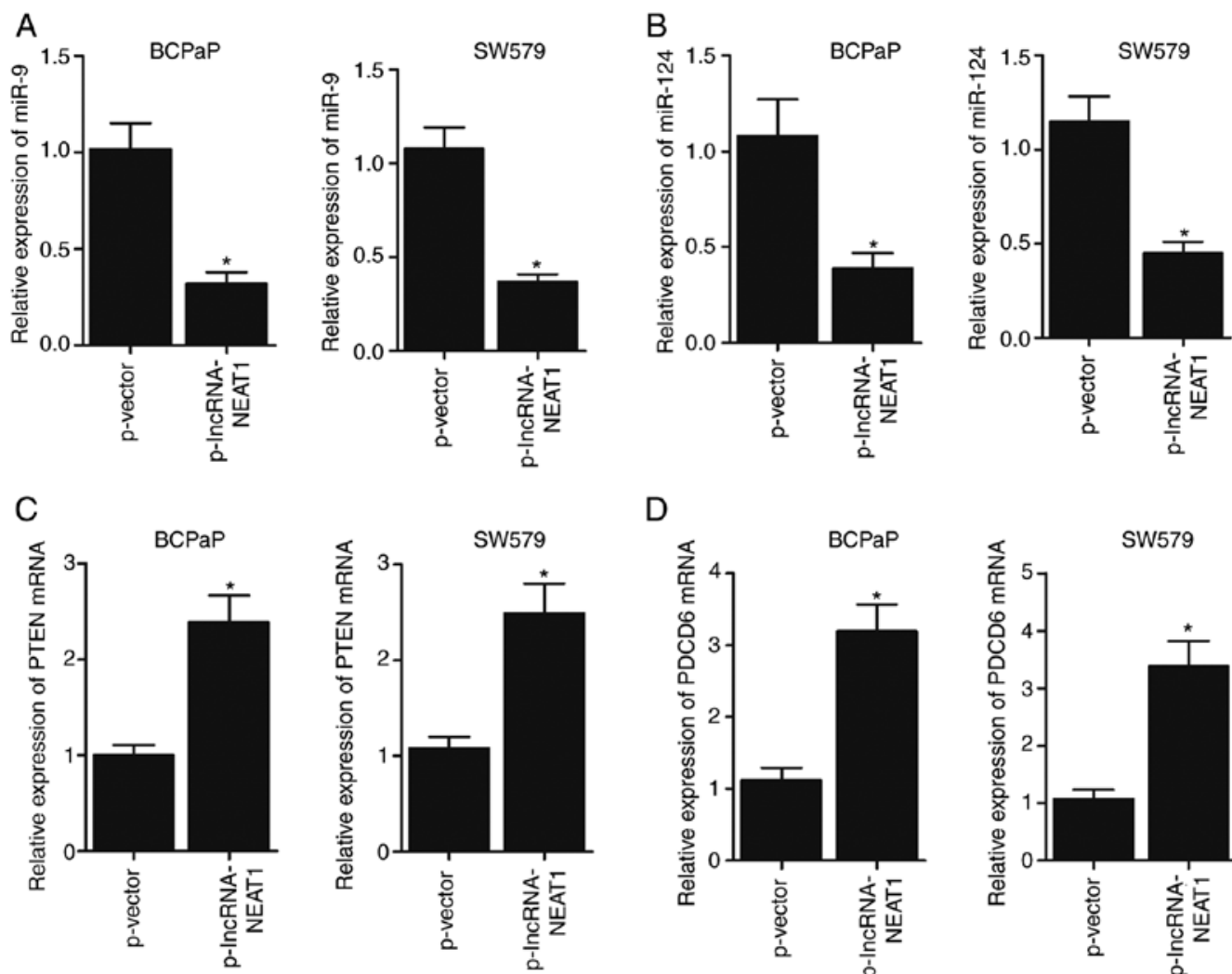

D
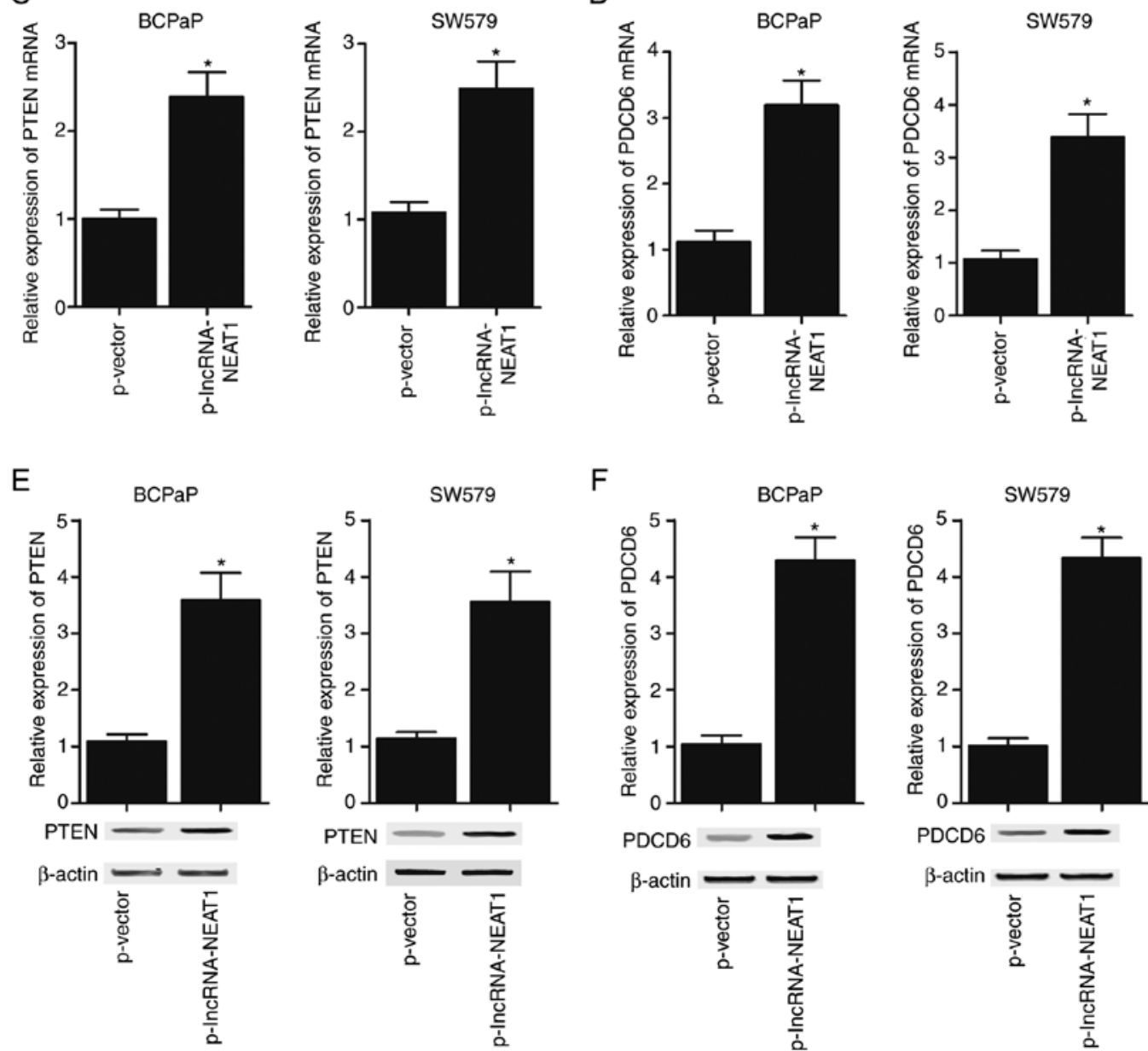

Figure 6. Upregulation of NEAT1 affects the regulatory relationship among NEAT1, miR-124, miR-9, PTEN and PDCD6 determined using reverse transcription-quantitative PCR and western blot analysis in BCPaP and SW579 cells. (A) Transfection with the NEAT1 overexpression vector downregulated miR-9 expression compared with that in the empty vector-transfected cells. (B) Transfection with the NEAT1 overexpression vector downregulated miR-124 expression compared with that in vector-transfected cells. (C) PTEN mRNA expression in BCPaP and SW579 cells was upregulated after transfection with the NEAT1 overexpression vector compared with that in the control cells. (D) PDCD6 mRNA expression in BCPaP and SW579 cells was upregulated after transfection with the NEAT1 overexpression vector compared with that in the control cells. (E) The protein level of PTEN was upregulated following transfection with the NEAT1 overexpression vector compared with that in the empty vector-transfected cells. (F) The protein level of PDCD6 was upregulated following transfection with the NEAT1 overexpression vector compared with that in the empty vector-transfected cells. ${ }^{*}<<0.05$ vs. vector. NEAT1, nuclear enriched abundant transcript 1; p-vector, plasmid-vector; miR, microRNA; PDCD6, programmed cell death protein 6.

transcriptional target of $\mathrm{p} 53$. The suppression of NEAT1 induced by 553 attenuated the inhibitory effect of p53 on cancer cell proliferation and modulated gene transactivation, indicating that NEAT1 may contribute to the potential tumour-suppressor function of p53 (42). In the present study, the diagnostic value of NEAT1, miR-124, and miR-9 was assessed using ROC analysis. The results demonstrated that the AUC values of NEAT1 were higher compared with those of miR-124 and miR-9 in peripheral blood samples and thyroid tissues. Therefore, the results of the present study suggested that NEAT1 could be used as a biomarker to differentiate between benign and malignant thyroid nodules.

In conclusion, the results of the present study demonstrated the use of NEAT1 as a potential biomarker to differentiate 

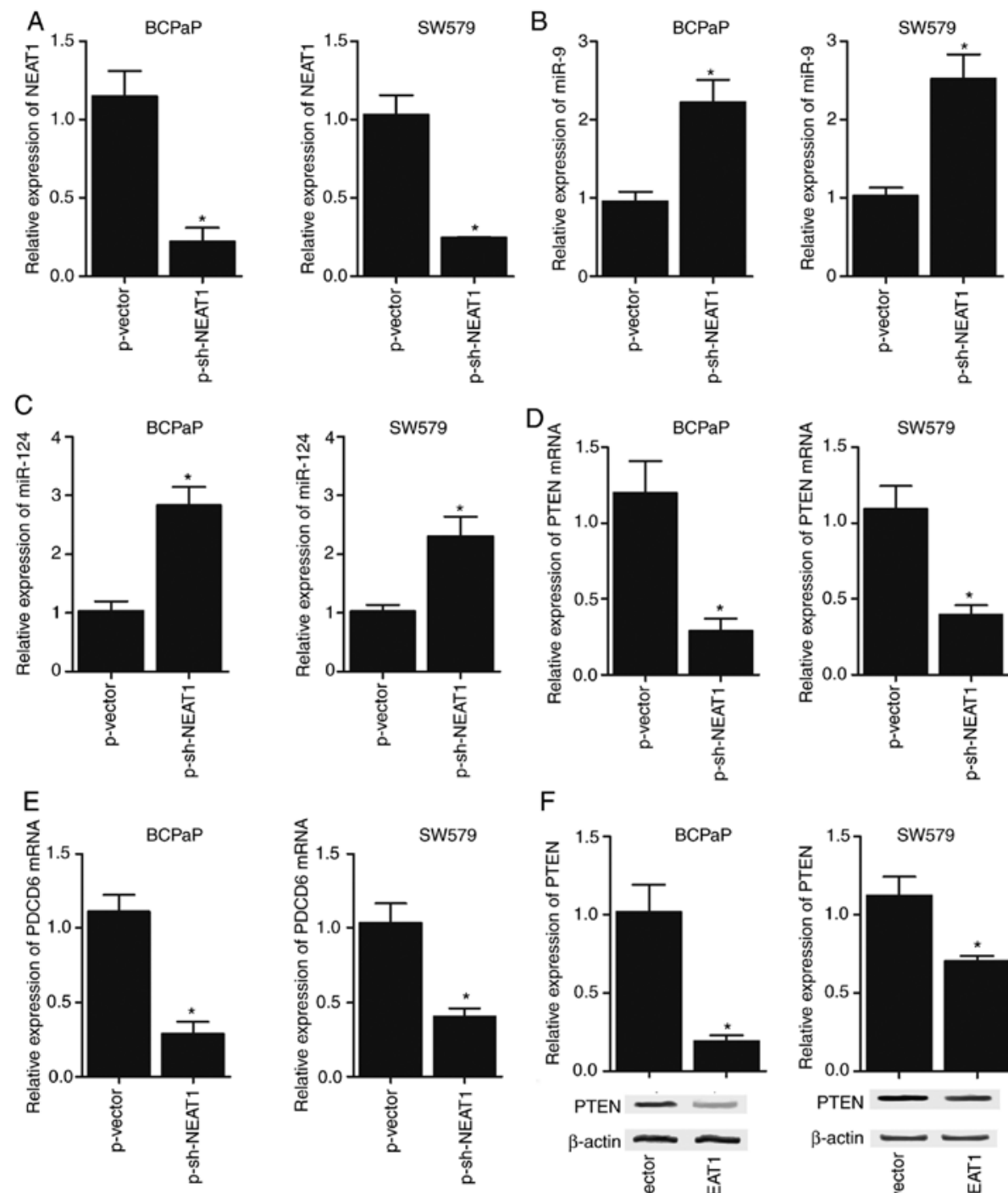

$\mathrm{F}$
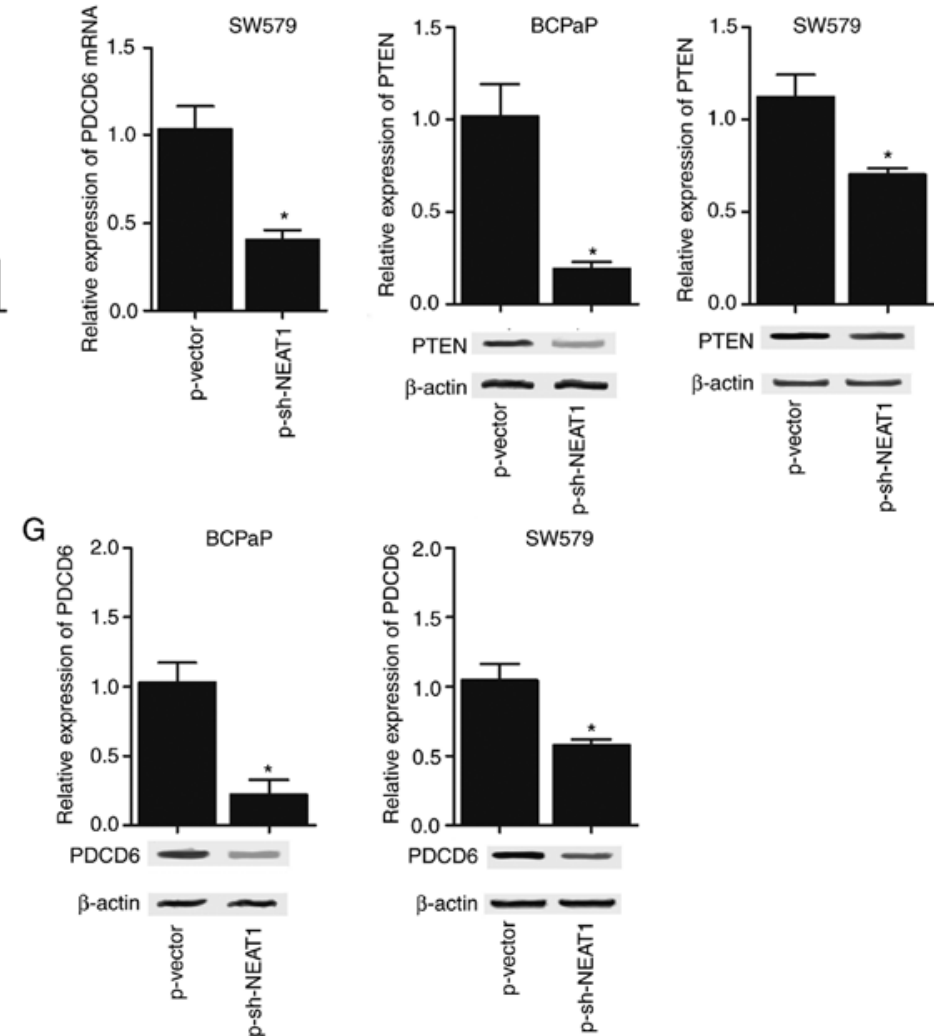

Figure 7. Downregulation of NEAT1 affects the regulatory relationship among NEAT1, miR-124, miR-9, PTEN and PDCD6 determined using reverse transcription-quantitative PCR and western blot analysis in BCPaP and SW579 cells. (A) sh-NEAT1 reduced NEAT1 expression. (B) Transfection with sh-NEAT1 upregulated miR-9 expression compared with that in cells transfected with the control vector. (C) Transfection with sh-NEAT1 upregulated miR-124 expression compared with that in cells transfected with the control vector. (D) PTEN mRNA expression in cells was decreased after transfection with sh-NEAT1 compared with that in cells transfected with the control vector. (E) PDCD6 mRNA expression in cells was reduced after transfection with sh-NEAT1 compared with that in cells transfected with the control vector. (F) Protein expression of PTEN was downregulated following transfection with sh-NEAT1 compared with that in cells transfected with the control vector. (G) Protein expression of PDCD6 was downregulated following transfection with sh-NEAT1 compared with that in cells transfected with the control vector. " $\mathrm{P}<0.05$ vs. p-vector. NEAT1, nuclear enriched abundant transcript 1 ; p-vector, plasmid-vector; miR, microRNA; PDCD6, programmed cell death protein 6.

benign and malignant thyroid nodules. In addition, NEAT1 may function as a sponge of miR-9 and miR-124. The present study also identified PTEN and PDCD6 as possible targets of miR-9 and miR-124, respectively. 


\section{Acknowledgements}

Not applicable.

\section{Funding}

No funding was received.

\section{Availability of data and materials}

The datasets used and/or analyzed during the current study are available from the corresponding author on reasonable request.

\section{Authors' contributions}

LZ designed and supervised the study, participated the experiments and drafted the manuscript. NZ participated the experiments and analyzed the data. PZ performed literature research, drafted and reviewed the manuscript. All authors read and approved the final manuscript.

\section{Ethics approval and consent to participate}

The present study was approved by the Ethics Committee of Shangluo Central Hospital (Shangluo, China), and all subjects provided written informed consent.

\section{Patient consent for publication}

Not applicable.

\section{Competing interests}

The authors declare that they have no competing interests.

\section{References}

1. Shaheen Z, BenHamed NA and Elhamdani RM: MON-436 cribriform-morular variant of papillary thyroid carcinoma: A case report. J Endocr Soc 4 (Suppl 1): MON-436, 2020.

2. Frates MC, Benson CB, Charboneau JW, Cibas ES, Clark OH, Coleman BG, Cronan JJ, Doubilet PM, Evans DB, Goellner JR, et al: Management of thyroid nodules detected at US: Society of radiologists in ultrasound consensus conference statement. Ultrasound Q 22: 231-240, 2006.

3. Papini E, Guglielmi R, Bianchini A, Crescenzi A, Taccogna S, Nardi F, Panunzi C, Rinaldi R, Toscano V and Pacella CM: Risk of malignancy in nonpalpable thyroid nodules: Predictive value of ultrasound and color-Doppler features. J Clin Endocrinol Metab 87: 1941-1946, 2002.

4. Frates MC, Benson CB, Doubilet PM, Kunreuther E, Contreras M, Cibas ES, Orcutt J, Moore FD Jr, Larsen PR, Marqusee E and Alexander EK: Prevalence and distribution of carcinoma in patients with solitary and multiple thyroid nodules on sonography. J Clin Endocrinol Metab 91: 3411-3417, 2006.

5. Yassa L, Cibas ES, Benson CB, Frates MC, Doubilet PM, Gawande AA, Moore FD Jr, Kim BW, Nosé V, Marqusee E, et al: Long-term assessment of a multidisciplinary approach to thyroid nodule diagnostic evaluation. Cancer 111: 508-516, 2007.

6. Haugen BR: 2015 American thyroid association management guidelines for adult patients with thyroid nodules and differentiated thyroid cancer: What is new and what has changed? Cancer 123: 372-381, 2017.

7. Wang CC, Friedman L, Kennedy GC, Wang H, Kebebew E, Steward DL, Zeiger MA, Westra WH, Wang Y, Khanafshar E, et al: A large multicenter correlation study of thyroid nodule cytopathology and histopathology. Thyroid 21: 243-251, 2011.
8. Maher B: ENCODE: The human encyclopedia. Nature 489: 46-48, 2012.

9. Dianatpour A and Ghafouri-Fard S: Long non-coding RNA expression intersecting cancer and spermatogenesis: A systematic review. Asian Pac J Cancer Prev 18: 2601-2610, 2017.

10. He JH, Han ZP and Li YG: Association between long non-coding RNA and human rare diseases (Review). Biomed Rep 2: 19-23, 2014.

11. Maruyama R, Suzuki H, Yamamoto E, Imai K and Shinomura Y: Emerging links between epigenetic alterations and dysregulation of noncoding RNAs in cancer. Tumour Biol 33: 277-285, 2012.

12. Mercer TR, Dinger ME and Mattick JS: Long non-coding RNAs: Insights into functions. Nat Rev Genet 10: 155-159, 2009.

13. Hu YC, Wang AM, Lu JK, Cen R and Liu LL: Long noncoding RNA HOXD-AS1 regulates proliferation of cervical cancer cells by activating Ras/ERK signaling pathway. Eur Rev Med Pharmacol Sci 21: 5049-5055, 2017.

14. Adriaens C and Marine JC: NEAT1-containing paraspeckles: Central hubs in stress response and tumor formation. Cell Cycle 16: 137-138, 2017.

15. Choudhry $\mathrm{H}$ and Mole DR: Hypoxic regulation of the noncoding genome and NEAT1. Brief Funct Genomics 15: 174-185, 2016.

16. Xie Q, Lin S, Zheng M, Cai Q and Tu Y: Long noncoding RNA NEAT1 promotes the growth of cervical cancer cells via sponging miR-9-5p. Biochem Cell Biol 97: 100-108, 2019.

17. Ma Y, Liu L, Yan F, Wei W, Deng J and Sun J: Enhanced expression of long non-coding RNA NEAT1 is associated with the progression of gastric adenocarcinomas. World J Surg Oncol 14: 41, 2016.

18. Gao C, Zhang J, Wang Q and Ren C: Overexpression of lncRNA NEAT1 mitigates multidrug resistance by inhibiting ABCG2 in leukemia. Oncol Lett 12: 1051-1057, 2016.

19. Lu E, Su J, Zeng W and Zhang C: Enhanced miR-9 promotes laryngocarcinoma cell survival via down-regulating PTEN. Biomed Pharmacother 84: 608-613, 2016.

20. Cheng $\mathrm{N}$ and Guo $\mathrm{Y}$ : Long noncoding RNA NEAT1 promotes nasopharyngeal carcinoma progression through regulation of miR-124/NF- $\kappa$ B pathway. Onco Targets Ther 10: 5843-5853, 2017.

21. Zhang L, Chen X, Liu B and Han J: MicroRNA-124-3p directly targets PDCD6 to inhibit metastasis in breast cancer. Oncol Lett 15: 984-990, 2018.

22. Yu S, Liu X, Zhang Y, Li J, Chen S, Zheng H, Reng R, Zhang $C$, Chen $J$ and Chen L: Circulating microRNA124-3p, microRNA9-3p and microRNA196b-5p may be potential signatures for differential diagnosis of thyroid nodules. Oncotarget 7: 84165-84177, 2016

23. Lei H, Gao Y and Xu X: LncRNA TUG1 influences papillary thyroid cancer cell proliferation, migration and EMT formation through targeting miR-145. Acta Biochim Biophys Sin (Shanghai) 49: 588-597, 2017.

24. Bartolazzi A, Sciacchitano S and D'Alessandria C: Galectin-3: The impact on the clinical management of patients with thyroid nodules and future perspectives. Int J Mol Sci 19: 445, 2018.

25. Zhang YZ, Xu T, Gong HY, Li CY, Ye XH, Lin HJ, Shen MP, Duan Y, Yang T and Wu XH: Application of high-resolution ultrasound, real-time elastography, and contrast-enhanced ultrasound in differentiating solid thyroid nodules. Medicine (Baltimore) 95: e5329, 2016.

26. Livak KJ and Schmittgen TD: Analysis of relative gene expression data using real-time quantitative PCR and the 2(-Delta Delta C(T)) method. Methods 25: 402-408, 2001.

27. Morselli E, Galluzzi L, Kepp O, Vicencio JM, Criollo A, Maiuri MC and Kroemer G: Anti- and pro-tumor functions of autophagy. Biochim Biophys Acta 1793: 1524-1532, 2009.

28. Su CH, Chen LJ, Liao JF and Cheng JT: Increase of phosphatase and tensin homolog by silymarin to inhibit human pharynx squamous cancer. J Med Food 16: 778-784, 2013.

29. Eng C: PTEN: One gene, many syndromes. Hum Mutat 22: 183-198, 2003.

30. Hobert JA, Embacher R, Mester JL, Frazier TW II and Eng C: Biochemical screening and PTEN mutation analysis in individuals with autism spectrum disorders and macrocephaly. Eur J Hum Genet 22: 273-276, 2014.

31. Vito $P$, Lacanà $E$ and $D$ 'Adamio $L$ : Interfering with apoptosis: $\mathrm{Ca}(2+)$-binding protein ALG-2 and Alzheimer's disease gene ALG-3. Science 271: 521-525, 1996.

32. Maki M, Narayana SV and Hitomi K: A growing family of the $\mathrm{Ca}^{2+}$-binding proteins with five EF-hand motifs. Biochem J 328: 718-720, 1997. 
33. Ohkouchi S, Nishio K, Maeda M, Hitomi K, Adachi $\mathrm{H}$ and Maki $M$ : Identification and characterization of two penta-EF-hand $\mathrm{Ca}(2+)$-binding proteins in Dictyostelium discoideum. J Biochem 130: 207-215, 2001.

34. Su D, Xu H, Feng J, Gao Y, Gu L, Ying L, Katsaros D, Yu H, Xu S and Qi M: PDCD6 is an independent predictor of progression free survival in epithelial ovarian cancer. J Transl Med 10: 31, 2012.

35. la Cour JM, Høj BR, Mollerup J, Simon R, Sauter G and Berchtold MW: The apoptosis linked gene ALG-2 is dysregulated in tumors of various origin and contributes to cancer cell viability. Mol Oncol 1: 431-439, 2008.

36. Jiang X, Zhou Y, Sun AJ and Xue JL: NEAT1 contributes to breast cancer progression through modulating miR-448 and ZEB1. J Cell Physiol 233: 8558-8566, 2018.

37. Zhao D, Zhang Y, Wang N and Yu N: NEAT1 negatively regulates miR-218 expression and promotes breast cancer progression. Cancer Biomark 20: 247-254, 2017.

38. Han D, Wang J and Cheng G: LncRNA NEAT1 enhances the radio-resistance of cervical cancer via miR-193b-3p/CCND1 axis. Oncotarget 9: 2395-2409, 2017.
39. Souquere S, Beauclair G, Harper F, Fox A and Pierron G: Highly ordered spatial organization of the structural long noncoding NEAT1 RNAs within paraspeckle nuclear bodies. Mol Biol Cell 21: 4020-4027, 2010.

40. Yu X, Li Z, Zheng H, Chan MT and Wu WK: NEAT1: A novel cancer-related long non-coding RNA. Cell Prolif 50: e12329, 2017.

41. Chai Y, Liu J, Zhang Z and Liu L: HuR-regulated lncRNA NEAT1 stability in tumorigenesis and progression of ovarian cancer. Cancer Med 5: 1588-1598, 2016.

42. Idogawa M, Ohashi T, Sasaki Y, Nakase H and Tokino T: Long non-coding RNA NEAT1 is a transcriptional target of p53 and modulates p53-induced transactivation and tumor-suppressor function. Int J Cancer 140: 2785-2791, 2017.

This work is licensed under a Creative Commons

Attribution-NonCommercial-NoDerivatives 4.0 International (CC BY-NC-ND 4.0) License. 